\title{
Indicatori per la valutazione a scala geografica regionale dell'efficacia dello Standard 1.1 di condizionalità (solchi acquai temporanei)
}

\author{
Paolo Bazzoffi \\ CREA-ABP, Centro di Ricerca per l'Agrobiologia e la Pedologia, Consiglio per la Ricerca in Agricoltura e l'Analisi dell'Economia \\ Agraria, Firenze, Italia
}

Autore corrispondente: Paolo Bazzoffi
E-mail: paolo.bazzoffi@crea.gov.it

Parole chiave: Indicatori; condizionalità; standard; erosione; solchi acquai.

Lavoro svolto nell'ambito del Progetto M0.NA.C0. (Rete di monitoraggio nazionale dell'efficacia ambientale della condizionalità e del differenziale di competitività da essa indotto a carico delle imprese agricole) finanziato dal Ministero delle Politiche Agricole, Alimentari e Forestali (MiPAAF) nell'ambito del Programma Rete Rurale Nazionale nel contesto dell'Azione 1.2.2 "Laboratori interregionali per lo sviluppo" del Programma Operativo denominato "Rete Rurale Nazionale 2007 - 2013.

Paolo Bazzoffi: Dirigente di Ricerca CREA-ABP, Coordinatore del progetto MO.NA.CO.
CCopyright P. Bazzoffi, 2015

Licenziatario PAGEPress, Italy

Italian Journal of Agronomy 2015; 10(s1):718

doi:10.4081/ija.2015.718

Questo articolo è distribuito secondo i termini della licenza Noncommercial Creative Commons Attribution (by-nc 3.0) che permette qualsiasi uso non commerciale, la distribuzione e la riproduzione con qualsiasi mezzo, a condizione che l'autore (autori) originale(i) e la fonte siano accreditati.

\section{Premessa}

Il Reg. di esecuzione (CE) N. 808/2014 (All. V - Questionario valutativo comune per lo sviluppo rurale) impone che nelle relazioni annuali sull'attuazione (RAE) presentate nel 2017 e nel 2019, nonché nella relazione di valutazione ex post, debba essere fornita risposta all'Aspetto specifico 4C: "in che misura i PSR hanno contribuito alla prevenzione dell'erosione dei suoli e a una migliore gestione degli stessi?".

Il "Valutatore Indipendente", chiamato ad effettuare la valutazione del PSR sotto la responsabilità dell'Autorità di Gestione (art. 84, paragrafo 4, del Reg. (CE) n. 1698/2005), per quantificare correttamente l'efficacia ambientale delle misure di Sviluppo Rurale aventi valenza nel diminuire l'erosione del suolo (in modo particolare le azioni della Misura 10 dei PSR 2014-2020), dovrà valutare preventivamente l'effetto della baseline, ovvero l'efficacia dello Standard 1.1 (impegno a) di condizionalità. Una volta effettuata questa valutazione, sarà possibile determinare l'efficacia aggiuntiva delle diverse misure dell'agroambiente finalizzate alla Priorità 4.c - Prevenire l'erosione del suolo e migliorarne la gestione.

Per la valutazione dell'efficacia dei solchi acquai temporanei nella diminuzione del rischio di erosione è consigliabile che il Valutatore Indipendente utilizzi una modellistica di facile applicazione. Nel presente lavoro si propongono due modelli di regressione che consentono di stimare l'erosione del suolo in presenza dei solchi acquai (trattamento fattuale, F) 0 in assenza di solchi acquai temporanei (trattamento controfattuale, CF), nell'ipotesi di utilizzazione del suolo a cereale a paglia autunno-vernino. Nel caso di colture diverse, facendo variare i valori medi annui del fattore colturale e del fattore pratiche conservative dell'equazione RUSLE è possibile valutare l'efficacia di contenimento dell'erosione in relazione sia agli impegni di condizionalità sia a quelli della M10 dei PSR, finalizzati anche alla conservazione del suolo.

\section{I modelli}

Come universalmente noto, l'equazione universale per le perdite di suolo USLE (Universal Soil Loss Equation; Wischmeier e Smith, 1978) è il modello più largamente usato nel mondo negli studi sull'erosione a differenti scale geografiche. Si tratta di un modello semplice di natura empirica adatto alla stima, come media di lungo periodo, dell'erosione del suolo.

Nella formulazione originaria l'equazione universale della perdita di suolo si presenta nel seguente modo:

$$
\mathrm{A}=\mathrm{R} * \mathrm{~K} * \mathrm{LS} * \mathrm{C} * \mathrm{P}
$$

dove:

A rappresenta le perdite annue di suolo per unità di superficie (nel sistema metrico decimale vengono espresse in $\mathrm{Mg} \mathrm{ha}^{-1}$ anno- $^{-1}$ );

$\mathrm{R}$ indica l'aggressività, 0 meglio, il potere erosivo delle precipitazio$\mathrm{ni}$;

K rappresenta "l'erodibilità" del suolo, cioè la sua attitudine ad essere eroso dall'aggressività della pioggia;

- LS è un fattore legato alla situazione topografica del versante e deriva dalla combinazione della lunghezza (sottofattore L) e della pendenza (sottofattore $S$ );

- C è un fattore connesso con il tipo di copertura vegetale del suolo;

$P$ è un parametro che tiene conto delle eventuali tecniche di regimazione idrica e/o delle pratiche colturali eventualmente messe in atto per contrastare il fenomeno erosivo.

Sebbene l'equazione abbia molte limitazioni, essa è largamente usata per la sua semplicità di applicazione e per la sua robustezza (Desmet e Govers, 1996); soprattutto nella sua versione revisionata RUSLE (Renard et al., 1997).

La RUSLE è stata applicata dalla maggior parte delle Regioni Italiane per la produzione di mappe del rischio di erosione potenziale e reale ai fini dell'attuazione delle politiche di sviluppo rurale (PSR); pertanto si ritiene opportuno non utilizzare altri modelli previsionali, come ad 
esempio il modello PESERA (Gobin et al.,1999) che, pur indicato in Europa come modello di riferimento, determinerebbe una discrepanza nell'interpretazione dei risultati.

\section{Applicazione del modello RUSLE in 60 aree del controllo AGEA 2009}

In un precedente lavoro, realizzato nel progetto EFFICOND (Bazzoffi et al., 2011), il modello RUSLE è stato applicato nella valutazione comparativa del rischio di erosione su aree campione in presenza 0 in assenza dei solchi acquai, così come prescritti dalla Norma $1.1_{\text {(impegno a). }}$.

Al fine di un'indagine significativa si sono scelte 60 aree cerealicole (casi studio) nelle aree campione del controllo AGEA 2009 (Figura 1). Tali aree sono caratterizzate da un notevole rischio di erosione, in relazione al suolo, alla morfologia e al clima.

Le immagini satellitari ad alta risoluzione sono state messe a disposizione dal AGEA-SIN e l'analisi è stata condotta in ambiente ArcGIS applicando la RUSLE secondo una metodologia originale messa a punto per la presente indagine. La nuova metodologia simula la presenza dei solchi acquai sulle aree campione mediante l'affossamento del modello digitale delle quote in corrispondenza delle curve di livello distanziate $80 \mathrm{~m}$ le une dalle altre.

Gli strati usati nell'applicazione della metodologia sono: 1) Modello Digitale delle quote (DEM) su territorio nazionale a risoluzione 20 metri con estensione Fuso 32 (MATTM-Ministero dell'Ambiente e della Tutela del Territorio e del Mare); 2) Raster dell'erosività della pioggia a maglia 75 m (P. Bazzoffi, comunicazione personale); 3) Strato vettoriale dell'erodibilità del suolo (dal progetto BADASUOLI; E. Costantini, comunicazione personale).

Ai fini della valutazione in oggetto, i fattori richiesti dalla USLE sono stati così determinati:

- fattore R: cartografia digitale in formato raster dell'erosività delle piogge con maglia di 75 m (P. Bazzoffi, comunicazione personale);

- fattore K: cartografia digitale vettoriale dell'erodibilità del suolo (progetto BADASUOLI, E. Costantini, comunicazione personale);

- fattore C: in assenza di informazioni dettagliate sull'uso del suolo nelle aree di studio, è stato posto pari a 0,12 , valore che si può considerare rappresentativo di un generico utilizzo a seminativi;

- fattore P: in assenza di specifiche informazioni dettagliate è stato posto pari a 1;

- fattore LS: a differenza dei precedenti, il fattore LS è direttamente influenzato dalla presenza dei solchi acquai temporanei previsti dalla Norma 1.1. Per tale motivo, il punto focale del presente lavoro è stato il calcolo del fattore LS in assenza di solchi acquai e ipotizzandone la presenza.

Rispetto alla formulazione originaria della USLE, attualmente si tende a calcolare il fattore LS in maniera più complessa e aderente alla realtà morfologica del processo di scorrimento superficiale. In particolare è molto utilizzato il concetto di "Flow Accumulation" (0 anche "Catchment Area", ovvero "Area del bacino sotteso") (Moore and Burch, 1986; Mitasova et al., 1995; Desmet e Govers, 1996).

Dalla formulazione di Mitasova, ampiamente utilizzata, si ricava il fattore LS nel seguente modo:

$\mathrm{LS}=(\text { FlowAccumulation } * \text { CellSize/22,13) })^{0,4}$ *

$\left((\sin (\right.$ Slope $\left.* 0,001745) / 0,09){ }^{1,4}\right) * 1,4$

\section{dove}

FlowAccumulation è calcolata con ArcHydro Tools di ArcGis 9.3;

CellSize indica la dimensione in metri della griglia del DEM utilizzato;

Slope è l'inclinazione della cella espressa in gradi.
I risultati ottenuti con la formulazione di Mitasova sono però poco accurati per pendenze dei versanti elevate. Per tale motivo si è preferito adottare la formulazione proposta da Nearing (1997), che, pur rimanendo concettualmente analoga a quella di Mitasova, se ne differenzia per il diverso metodo di calcolo del termine dell'equazione che considera l'inclinazione del pendio.

Utilizzando la formulazione di Nearing il fattore LS è così calcolato:

LS $=(\text { FlowAccumulation } * \text { CellSize/22,13) })^{0,4}$ *

$(1,5+17 /(1+\operatorname{Exp}(2,3-6,1 * \operatorname{Sin}([$ slope ] $* 3,14 / 180))))$

Per simulare la presenza di solchi acquai, equidistanti $80 \mathrm{~m}$ sul piano campagna, sono stati utilizzati gli strumenti di DEM Manipulation e Terrain Preprocessing presenti in ArcHydro Tools di ArcGis. Il risultato di questo processo è un nuovo DEM in cui è simulata la presenza dei solchi acquai sulle aree campione mediante l'affossamento di $1 \mathrm{~m}$ delle quote del modello digitale originale in corrispondenza delle curve di livello distanziate $80 \mathrm{~m}$ le une dalle altre. Per affossare il DEM a $20 \mathrm{~m}$ lungo le curve di livello è stato necessario un ricampionamento del DEM a 1 metro.

Per ricavare l'equidistanza delle curve di livello corrispondenti a una

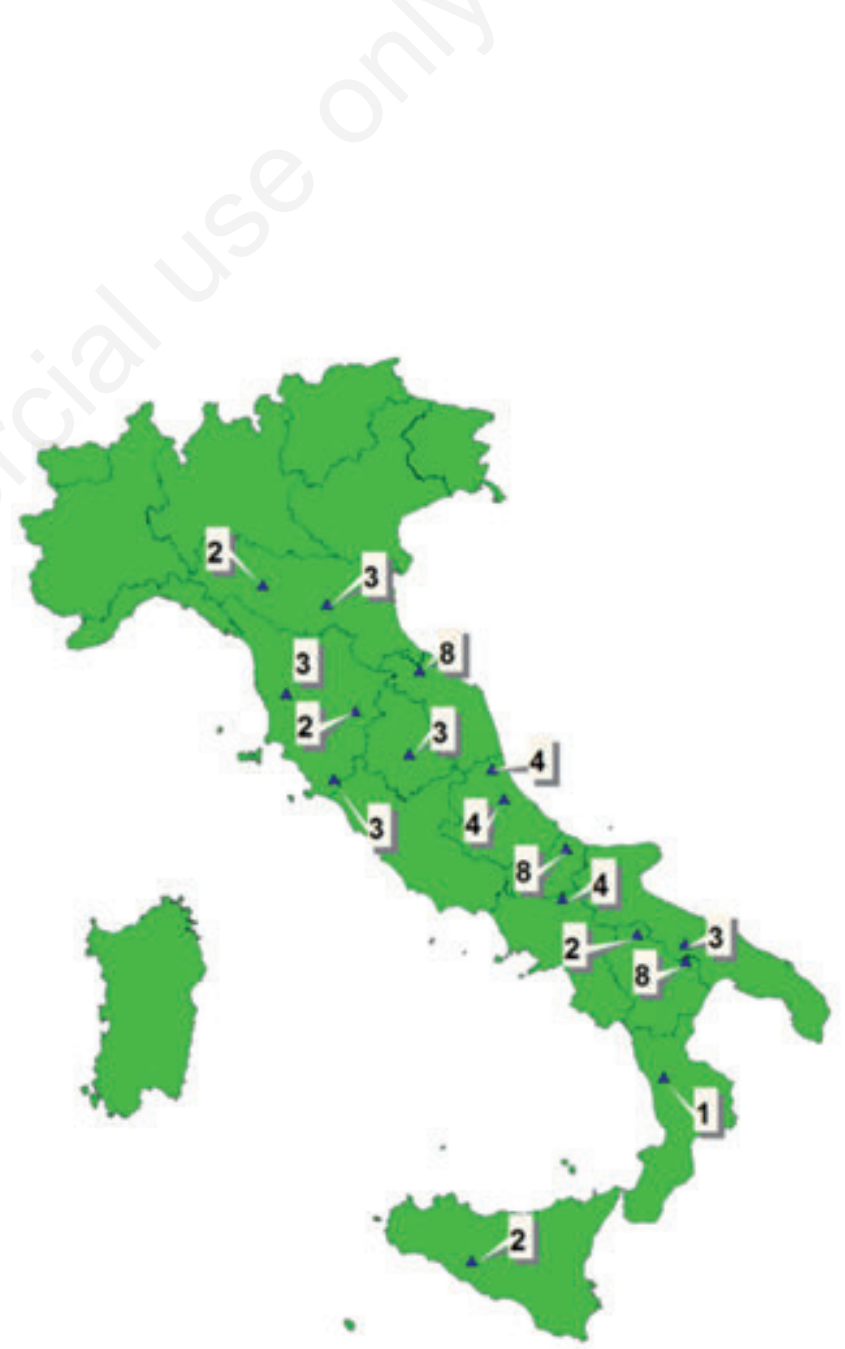

Figura 1. Localizzazione delle 60 aree-studio. I numeri indicano quante aree studio sono localizzate nei pressi dei siti indicati con i triangoli. 
distanza inclinata pari a 80 metri [in applicazione dello Standard $1.1_{\text {(impegno a) }}$ ] si è utilizzata la pendenza media dell'area in esame e tale valore si è utilizzato nella seguente formula:

Equidistanza curve $(\mathrm{m})=80 *(\operatorname{Sin}(\alpha / 180 * \pi))$

dove $\alpha$ è la pendenza media dell'area in gradi sessagesimali.

Utilizzando un foglio di calcolo Microsoft Excel la formula per ricavare l'equidistanza è la seguente:

$$
=80 *(\operatorname{SEN}(\mathrm{A} 2 / 180 * \mathrm{PI} . \mathrm{GRECO}()))
$$

In questo modo è stato possibile calcolare la perdita di suolo utilizzando il fattore LS in assenza di solchi acquai. Successivamente, mantenendo invariati gli altri fattori ed utilizzando il fattore LS ottenuto simulando la presenza di solchi acquai, si è ottenuto il valore di erosione in attuazione dello Standard $1.1_{\text {(impegno a). }}$.

Nella Figura 2 viene mostrata la comparazione del rischio di erosione su una delle aree studio, ottenuta mediante la procedura RUSLE applicata simulando la presenza o l'assenza dei solchi acquai distanziati $80 \mathrm{~m}$. Si è utilizzata la distanza di $80 \mathrm{~m}$ in quanto è la massima consentita dalla Norma 1.1.

Nella Tabella 1 vengono illustrati i valori di perdita di suolo stimati nelle aree di studio, in assenza e in presenza di solchi acquai temporanei. Complessivamente, nelle 60 aree di studio, la presenza dei solchi acquai temporanei farebbe diminuire l'erosione mediamente del $67 \% \mathrm{e}$ questo risultato è in accordo con il valore di riduzione dell'erosione osservata sulle parcelle idrologiche attrezzate a Vicarello e con rilievo UAV-GIS, rispettivamente dell' $84,4 \%$ e del 41,7\% (Bazzoffi, 2015; Bazzoffi et al., 2015).

Dall'applicazione del modello risulta che in 22 aree di studio le perdite di suolo in presenza di solchi rientrerebbero entro la soglia di tollerabilità di $6 \mathrm{Mg} \mathrm{ha}^{-1}$ anno $^{-1}$ fissato dall'OCSE(2001), in 17 risulterebbero comprese fra il limite OCSE e il limite USDA (ossia tra 6 e 11,2 Mg ha $^{-1}$ anno $^{-1}$ ) (Schertz e Nearing, 2005); nei restanti 21 casi le perdite di suolo, pur risultando superiori al limite USDA (11,2 $\mathrm{Mg} \mathrm{ha}^{-1}$ anno-1) non raggiungerebbero valori catastrofici (valore massimo $34,2 \mathrm{Mg} \mathrm{ha}^{-1}$ anno $\left.^{-1}\right)$.
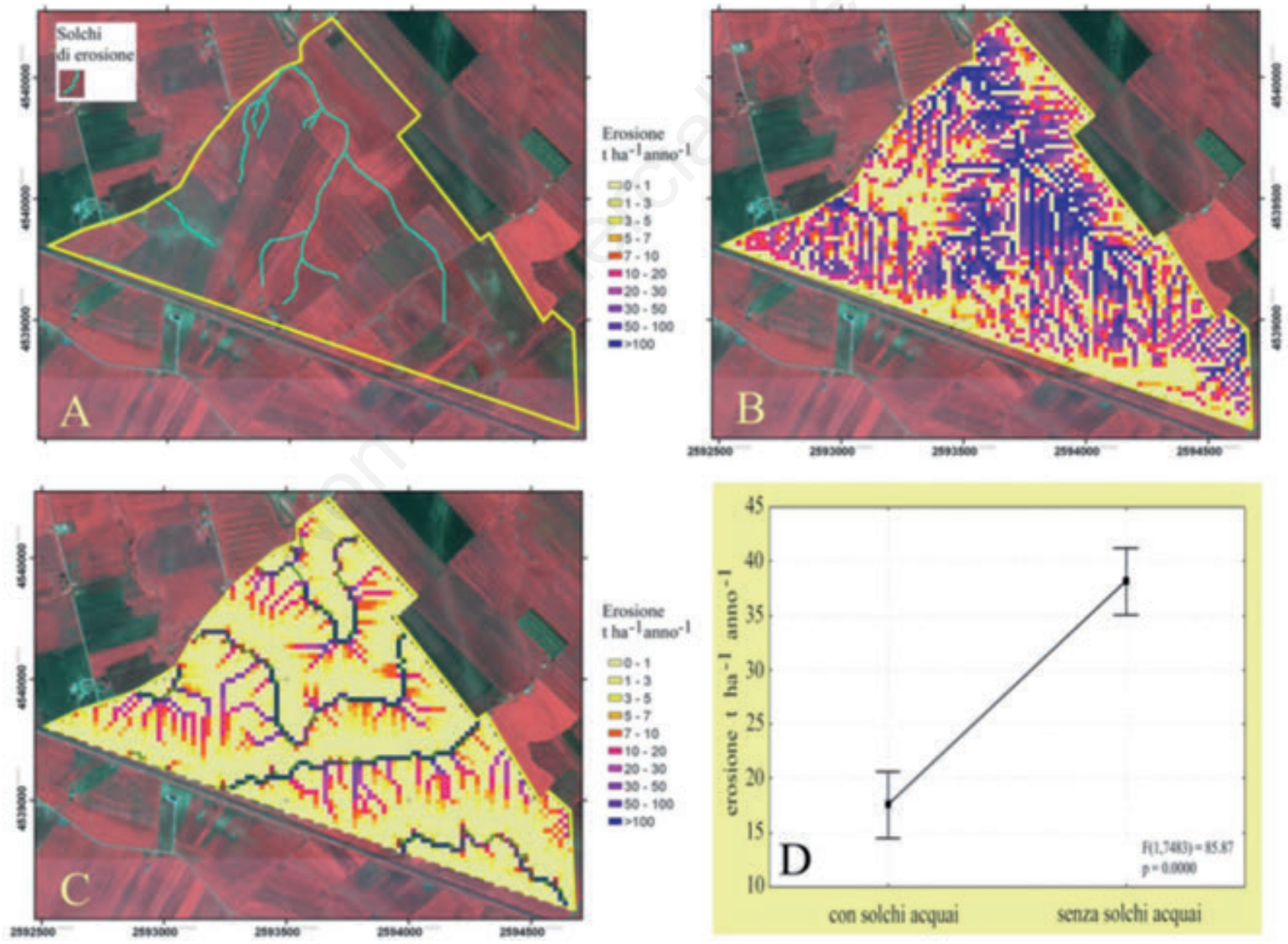

Figura 2. Comparazione del rischio di erosione su una delle aree campione del controllo AGEA 2009, ottenuta mediante la procedura RUSLE simulando l'assenza o la presenza dei solchi acquai distanziati $80 \mathrm{~m}$. A) Immagine satellitare con evidenziati i rigagnoli di erosione sviluppatisi nel periodo autunno-vernino 2008-2009. B) Rischio di erosione senza solchi acquai. C) Rischio di erosione con solchi acquai. D) Confronto statistico sui valori di erosione associati alle celle del raster. 
Tabella 1. Caratteristiche delle 60 aree di studio e risultati dell'applicazione del modello RUSLE in assenza e in presenza di solchi acquai temporanei distanziati $80 \mathrm{~m}$.

\begin{tabular}{|c|c|c|c|c|c|c|c|c|c|c|}
\hline $\begin{array}{l}\text { Provincia } \\
\text { e codice } \\
\text { dell'area } \\
\text { di studio }\end{array}$ & $\begin{array}{c}\text { Superficie } \\
\text { (ha) }\end{array}$ & $\begin{array}{l}\text { Fattore K } \\
\text { erodibilità } \\
\text { del suolo } \\
\left(\mathrm{Mg} \mathrm{ha} \mathrm{h} \mathrm{ha}^{-1}\right. \\
\left.\mathrm{mj}^{-1} \mathrm{~mm}^{-1}\right)\end{array}$ & $\begin{array}{c}\text { Fattore } \\
\text { R erosività } \\
\text { della pioggia } \\
\left(\mathrm{mj} \mathrm{mm} \mathrm{ha}^{-1}\right. \\
\mathrm{h}^{-1} \text { anno-1) }\end{array}$ & $\begin{array}{c}\text { Fattore } \\
\text { Colturale C } \\
\text { (adimensionale) }\end{array}$ & $\begin{array}{c}\text { Pendenza } \\
\text { media } \\
(\%)\end{array}$ & $\begin{array}{l}\text { Erosione } \\
\text { in assenza } \\
\text { di solchi } \\
\text { acquai } \\
\left(\mathrm{t} \mathrm{ha}{ }^{-1}\right. \\
\left.\text { anno-1 }^{-1}\right)\end{array}$ & $\begin{array}{l}\text { Erosione in } \\
\text { presenza } \\
\text { di solchi } \\
\text { acquai } \\
(\mathrm{t} \mathrm{ha-1} \\
\left.\text { anno-1 }^{-1}\right)\end{array}$ & $\begin{array}{c}\text { Ridurione } \\
\text { dell'erosione } \\
\text { per effetto } \\
\text { dei solchi } \\
\text { acquai } \\
\left(\mathrm{t} \mathrm{ha} \mathrm{a}^{-1} \text { anno-1) }\right.\end{array}$ & $\begin{array}{l}\text { Riduzione } \\
\text { dell'erosione } \\
\text { per effetto } \\
\text { dei solchi } \\
\text { acquai } \\
(\%)\end{array}$ & $\begin{array}{l}\text { Significatività } \\
\text { statistica della } \\
\text { diminuzione } \\
\text { dell'erosione } \\
\text { (con solchi } \\
\text { os senza solchi) }\end{array}$ \\
\hline AG_1 & 26,6 & 0,022 & 1656 & 0,12 & 19,5 & 30,8 & 6,2 & 24,7 & 80,0 & $* *$ \\
\hline AG_2 & 51,9 & 0,022 & 1618 & 0,12 & 13,8 & 19,1 & 7,8 & 11,3 & 59,3 & $* *$ \\
\hline AR_1 & 11,1 & 0,035 & 2041 & 0,12 & 9,2 & 20,7 & 6,6 & 14,0 & 67,9 & $* *$ \\
\hline AR_2 & 9,7 & 0,035 & 1951 & 0,12 & 6,4 & 15,3 & 5,2 & 10,0 & 65,7 & $* *$ \\
\hline BA_2 & 40,5 & 0,035 & 1155 & 0,12 & 4,7 & 6,0 & 1,7 & 4,3 & 71,4 & $* *$ \\
\hline BA_4 & 23,4 & 0,033 & 1091 & 0,12 & 4,3 & 4,5 & 1,3 & 3,2 & 71,0 & $* *$ \\
\hline BA_5 & 15,5 & 0,018 & 1235 & 0,12 & 8,5 & 4,7 & 1,7 & 3,0 & 63,4 & $* *$ \\
\hline BN_1 & 6,2 & 0,022 & 1900 & 0,12 & 11,3 & 12,2 & 5,3 & 7,0 & 57,0 & $* *$ \\
\hline BN_2 & 27,4 & 0,022 & 2457 & 0,12 & 9,8 & 29,6 & 8,5 & 21,2 & 71,4 & $* *$ \\
\hline BN_3 & 23,2 & 0,022 & 2566 & 0,12 & 13,0 & 33,2 & 13,6 & 19,6 & 59,0 & $* *$ \\
\hline BN_4 & 4,8 & 0,022 & 2010 & 0,12 & 11,2 & 12,1 & 5,2 & 6,9 & 57,0 & $* *$ \\
\hline BO_1 & 16,0 & 0,059 & 1736 & 0,12 & 15,3 & 52,3 & 18,7 & 33,6 & 64,3 & $* *$ \\
\hline BO_2 & 21,5 & 0,059 & 1669 & 0,12 & 10,8 & 31,4 & 13,8 & 17,6 & 55,9 & $* *$ \\
\hline BO_3 & 6,4 & 0,053 & 1596 & 0,12 & 16,6 & 45,3 & 10,6 & 34,7 & 76,6 & $* *$ \\
\hline CB_1 & 49,2 & 0,035 & 1032 & 0,12 & 10,2 & 14,8 & 4,1 & 10,7 & 72,2 & $* *$ \\
\hline CB_3 & 53,1 & 0,027 & 1090 & 0,12 & 10,6 & 11,7 & 3,0 & 8,6 & 74,0 & $* *$ \\
\hline CB_4 & 97,4 & 0,022 & 1013 & 0,12 & 16,1 & 17,7 & 4,5 & 13,2 & 74,7 & $* *$ \\
\hline CB_5 & 48,4 & 0,022 & 1107 & 0,12 & 7,5 & 7,6 & 2,0 & 5,6 & 74,1 & $* *$ \\
\hline $\mathrm{CB} \_6$ & 24,6 & 0,022 & 1150 & 0,12 & 11,7 & 12,6 & 3,7 & 8,9 & 70,5 & $* *$ \\
\hline CB_7 & 16,7 & 0,022 & 1445 & 0,12 & 12,6 & 13,9 & 5,2 & 8,6 & 62,2 & $* *$ \\
\hline CB_8 & 23,5 & 0,022 & 1643 & 0,12 & 13,5 & 21,1 & 7,6 & 13,6 & 64,1 & $* *$ \\
\hline CB_9 & 34,3 & 0,022 & 1670 & 0,12 & 7,6 & 8,7 & 2,3 & 6,4 & 73,1 & $* *$ \\
\hline CS_1 & 4,8 & 0,034 & 2903 & 0,12 & 9,4 & 17,4 & 9,1 & 8,2 & 47,4 & $* *$ \\
\hline GR_1 & 19,9 & 0,035 & 2646 & 0,12 & 7,3 & 19,7 & 5,9 & 13,9 & 70,3 & $* *$ \\
\hline GR_2 & 22,1 & 0,039 & 2630 & 0,12 & 8,2 & 23,0 & 3,8 & 19,2 & 83,7 & $* *$ \\
\hline GR_3 & 16,7 & 0,047 & 2592 & 0,12 & 4,7 & 15,9 & 4,7 & 11,2 & 70,4 & $* *$ \\
\hline MT_1 & 9,4 & 0,036 & 1660 & 0,12 & 13,1 & 26,4 & 10,3 & 16,1 & 61,0 & $* *$ \\
\hline MT_2 & 32,0 & 0,040 & 1672 & 0,12 & 15,6 & 35,2 & 13,6 & 21,6 & 61,5 & $* *$ \\
\hline MT_3 & 50,3 & 0,059 & 1656 & 0,12 & 7,1 & 18,9 & 7,3 & 11,6 & 61,1 & $* *$ \\
\hline MT_4 & 9,9 & 0,052 & 1550 & 0,12 & 8,4 & 14,2 & 7,4 & 6,9 & 48,2 & $* *$ \\
\hline MT_5 & 12,8 & 0,035 & 1592 & 0,12 & 19,0 & 56,3 & 14,9 & 41,5 & 73,6 & $* *$ \\
\hline MT_6 & 11,6 & 0,018 & 1367 & 0,12 & 15,7 & 15,8 & 5,9 & 9,9 & 62,7 & $* *$ \\
\hline MT_7 & 70,1 & 0,033 & 1090 & 0,12 & 5,5 & 7,1 & 1,7 & 5,4 & 76,3 & $* *$ \\
\hline MT_9 & 27,3 & 0,035 & 1471 & 0,12 & 9,1 & 12,0 & 4,8 & 7,1 & 59,6 & $* *$ \\
\hline PE_1 & 6,5 & 0,035 & 1961 & 0,12 & 16,3 & 29,8 & 9,7 & 20,2 & 67,6 & $* *$ \\
\hline PE_2 & 10,8 & 0,047 & 1784 & 0,12 & 9,6 & 24,2 & 7,7 & 16,5 & 68,3 & $* *$ \\
\hline PE_3 & 45,0 & 0,039 & 1830 & 0,12 & 15,0 & 42,3 & 14,3 & 28,0 & 66,3 & $* *$ \\
\hline PE_4 & 5,9 & 0,035 & 2049 & 0,12 & 19,2 & 30,8 & 9,7 & 21,1 & 68,4 & $* *$ \\
\hline PG_1 & 17,1 & 0,059 & 2633 & 0,12 & 12,0 & 58,6 & 19,0 & 39,7 & 67,6 & $* *$ \\
\hline PG_2 & 18,4 & 0,059 & 2583 & 0,12 & 10,1 & 59,1 & 16,6 & 42,5 & 71,9 & $* *$ \\
\hline PG_3 & 22,1 & 0,059 & 2693 & 0,12 & 8,7 & 52,5 & 16,6 & 35,9 & 68,4 & $* *$ \\
\hline PI_1 & 42,7 & 0,035 & 2827 & 0,12 & 7,3 & 25,8 & 6,0 & 19,8 & 76,6 & $* *$ \\
\hline PI_2 & 39,7 & 0,035 & 2805 & 0,12 & 7,0 & 20,4 & 6,1 & 14,3 & 70,1 & $* *$ \\
\hline PI_3 & 54,3 & 0,035 & 2766 & 0,12 & 10,3 & 29,8 & 8,7 & 21,1 & 70,9 & $* *$ \\
\hline
\end{tabular}




\section{Indicatori per la valutazione a scala geografica regionale dell'efficacia dello Standard 1.1 di condizionalità (solchi acquai temporanei)}

I valori di erosione stimata per le 60 aree di studio sono stati messi in correlazione con le seguenti variabili di bacino:

Pendenza Media (\%)
Pendenza Max. (\%)

Fattore K (Mg ha h ha $\left.{ }^{-1} \mathrm{MJ}^{-1} \mathrm{~mm}^{-1}\right)$

Fattore R (MJ mm ha ${ }^{-1} \mathrm{~h}^{-1}$ year $^{-1}$ )

Superficie parcella (ha)

Si sono così ricavati due modelli (o indicatori) ad uso del valutatore indipendente, che consentono di stimare facilmente il rischio di erosione su seminativi (nell' ipotesi di coltivazione di un cereale a paglia autunno-vernino) in applicazione dello Standard 1.1 a (Fattuale, ovvero con esecuzione dei solchi acquai) e in caso di infrazione

Tabella 1. Continua dalla pagina precedente.

\begin{tabular}{|c|c|c|c|c|c|c|c|c|c|c|}
\hline $\begin{array}{l}\text { Provincia } \\
\text { e codice } \\
\text { dell'area } \\
\text { di studio }\end{array}$ & $\begin{array}{c}\text { Superficie } \\
\text { (ha) }\end{array}$ & $\begin{array}{c}\text { Fattore K } \\
\text { erodibilità } \\
\text { del suolo } \\
\left(\mathrm{Mg} \mathrm{ha} \mathrm{h} \mathrm{ha}^{-1}\right. \\
\left.\mathrm{mj}^{-1} \mathrm{~mm}^{-1}\right)\end{array}$ & $\begin{array}{c}\text { Fattore } \\
\text { R erosività } \\
\text { della pioggia } \\
\left(\mathrm{mj} m \mathrm{ha}^{-1}\right. \\
\left.\mathrm{h}^{-1} \mathrm{anno}^{-1}\right)\end{array}$ & $\begin{array}{c}\text { Fattore } \\
\text { Colturale C } \\
\text { (adimensionale) }\end{array}$ & $\begin{array}{l}\text { Pendenza } \\
\text { media } \\
(\%)\end{array}$ & $\begin{array}{c}\text { Erosione } \\
\text { in assenza } \\
\text { di solchi } \\
\text { acquai } \\
\left(\mathrm{t} \mathrm{ha}^{-1}\right. \\
\left.\text { anno }^{-1}\right)\end{array}$ & $\begin{array}{l}\text { Erosione in } \\
\text { presenza } \\
\text { di solchi } \\
\text { acquai } \\
\left(\mathrm{t} \mathrm{ha}^{-1}\right. \\
\left.\text { anno }^{-1}\right)\end{array}$ & $\begin{array}{c}\text { Riduzione } \\
\text { dell'erosione } \\
\text { per effetto } \\
\text { dei solchi } \\
\text { acquai } \\
\left(\mathrm{t} \mathrm{ha} \mathrm{a}^{-1} \text { anno-1) }\right.\end{array}$ & $\begin{array}{l}\text { Riduzione } \\
\text { dell'erosione } \\
\text { per effetto } \\
\text { dei solchi } \\
\text { acquai } \\
(\%)\end{array}$ & $\begin{array}{l}\text { Significatività } \\
\text { statistica della } \\
\text { diminurione } \\
\text { dell'erosione } \\
\text { (con solchi } \\
\text { us. senza solchi) }\end{array}$ \\
\hline
\end{tabular}

\begin{tabular}{|c|c|c|c|c|c|c|c|c|c|c|}
\hline PR_1 & 11,7 & 0,047 & 2556 & 0,12 & 12,1 & 56,8 & 20,6 & 36,2 & 63,7 & ** \\
\hline PR_2 & 15,5 & 0,047 & 2544 & 0,12 & 10,6 & 37,5 & 12,8 & 24,7 & 65,8 & ** \\
\hline PU_1 & 13,7 & 0,053 & 2654 & 0,12 & 17,2 & 71,3 & 21,5 & 49,8 & 69,9 & ** \\
\hline PU_2 & 6,5 & 0,035 & 2821 & 0,12 & 17,9 & 46,9 & 13,1 & 33,8 & 72,1 & ** \\
\hline PU_3 & 17,1 & 0,035 & 2824 & 0,12 & 20,1 & 72,7 & 19,6 & 53,1 & 73,1 & ** \\
\hline PU_4 & 11,6 & 0,035 & 2761 & 0,12 & 25,5 & 89,2 & 34,2 & 55,0 & 61,6 & $* *$ \\
\hline PU_5 & 7,3 & 0,059 & 2745 & 0,12 & 18,0 & 118,3 & 30,2 & 88,1 & 74,5 & ** \\
\hline PU_6 & 3,3 & 0,043 & 2753 & 0,12 & 13,0 & 26,8 & 14,7 & 12,1 & 45,2 & ** \\
\hline PU_7 & 26,1 & 0,059 & 2736 & 0,12 & 20,2 & 114,4 & 27,9 & 86,5 & 75,6 & ** \\
\hline PU_8 & 25,1 & 0,058 & 2714 & 0,12 & 18,6 & 135,3 & 31,3 & 103,9 & 76,8 & ** \\
\hline PZ_1 & 112,1 & 0,035 & 1490 & 0,12 & 3,6 & 9,0 & 2,1 & 6,9 & 77,1 & ** \\
\hline PZ_2 & 110,0 & 0,035 & 1479 & 0,12 & 4,4 & 10,9 & 2,0 & 8,9 & 81,9 & ** \\
\hline TE_1 & 5,4 & 0,047 & 1747 & 0,12 & 9,7 & 18,9 & 7,4 & 11,4 & 60,5 & ** \\
\hline TE_2 & 11,9 & 0,035 & 1713 & 0,12 & 21,1 & 44,0 & 19,6 & 24,5 & 55,5 & ** \\
\hline TE_3 & 19,4 & 0,035 & 1703 & 0,12 & 14,9 & 35,7 & 12,8 & 22,9 & 64,2 & ** \\
\hline TE_4 & 13,0 & 0,035 & 1700 & 0,12 & 10,8 & 24,6 & 6,8 & 17,8 & 72,3 & ** \\
\hline
\end{tabular}

Tabella 2. Riepilogo della regressione e analisi della varianza per il modello Fattuale (con solchi acquai).

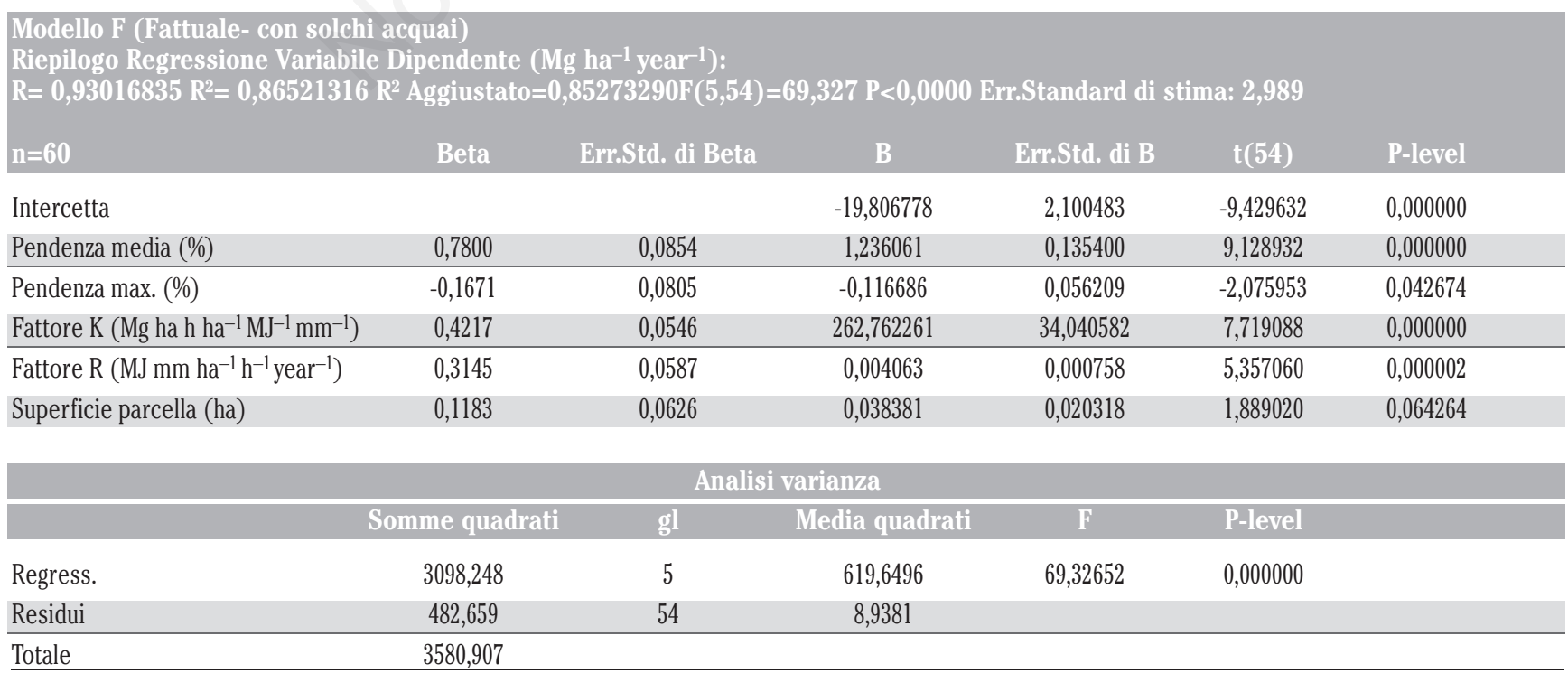


(Controfattuale, ovvero senza esecuzione dei solchi acquai).

Il due modelli sono i seguenti:

MODELLO F (Fattuale - con solchi acquai)

t ha ${ }^{-1}$ anno $^{-1}=-19,807+1,236 *$ Pend.Media (\%) $-0,1167 *$ Pend.

Massima (\%) $+262,76 * \mathrm{~K}+0,004063 * \mathrm{R}+0,038 *$ ha

MODELLO CF (ControFattuale - senza solchi acquai)

t ha ${ }^{-1}$ anno $^{-1}=-79,641+3,681 *$ Pend.Media (\%) (\%)-0,0653* Pend.

Massima $(\%)+978,77 * \mathrm{~K}+0,0145 * \mathrm{R}+0,074 *$ ha

(eq. 7)

Il riepilogo della regressione e l'analisi della varianza per i due modelli sono riportati nelle Tabelle 2 e 3 .

\section{Valutazione del potenziale di mitigazione dell'erosione degli impegni della Misura 10 (Pagamenti agro-climatico-ambientali) dei PSR 2014-2020}

La misura 10 dello Sviluppo Rurale 2014-2020 si articola in SOTTOMISURE le quali a loro volta si articolano in INTERVENTI che sono specifici per ciascuna Regione, Ciascun intervento contiene il dettaglio degli IMPEGNI (o azioni) che il beneficiario dei pagamenti compensativi si impegna ad adottare.

Diversi impegni della M10 impattano sulla copertura vegetale e sulla gestione del suolo, Ne deriva che la valutazione dell'efficacia della misura 10 nella protezione del suolo dall'erosione può essere fatta uti-

Tabella 3. Riepilogo della regressione e analisi della varianza per il modello Controfattuale (senza solchi acquai).

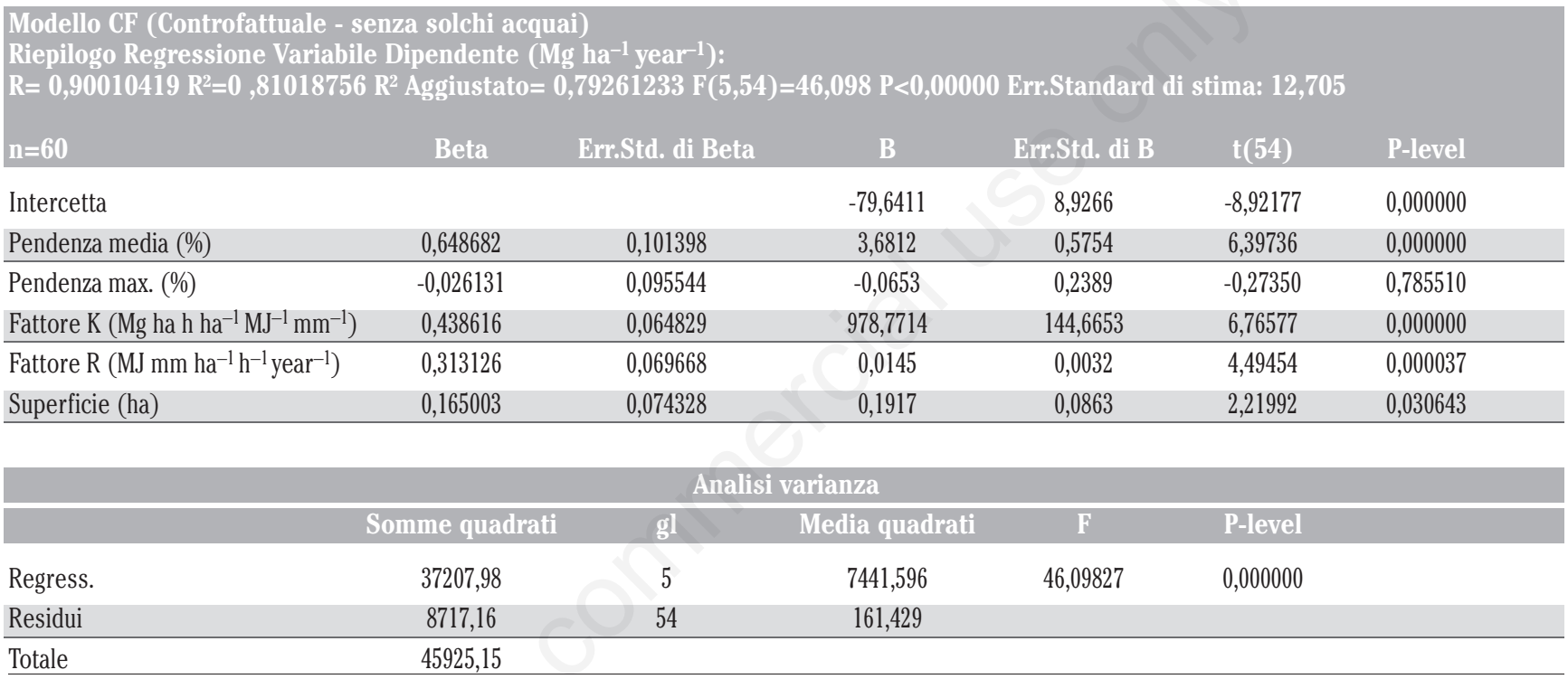

Tabella 4. Valori medi annui del fattore colturale C e del fattore P (pratiche conservative) dell'equazione RUSLE desunti dalla letteratura scientifica internazionale.

\section{Tlipo di coltura e gestione}

Suolo nudo

Suolo arato e preparato per la semina (senza residui di colture precedenti)

(Wischmeier e Smith, 1978)

Cereali a paglia e soia

Cereali a paglia (a semina autunnale o primaverile) e soia su quantità bassa di residui colturali

(Stone e Hilborn, 2012)

Soia e cereali a paglia (a semina autunnale o primaverile) su abbondanti residui colturali

(Stone e Hilborn, 2012)

1

Frutteti e altre arboree

Frutteti a vaso $\mathrm{m} 8 \mathrm{x} 8$ a chiome molto coprenti (valore per la parte sottochioma) (Rousseva, 2004)

Frutteti a vaso m 8x8 a chiome molto coprenti (valore medio per tutto il frutteto) (Rousseva, 2004)

Frutteti a palmetta (Rousseva, 2004)

Patata (e similari) V

Patata (e similari) dopo cereali a paglia con aratura estiva
0,12

0,09

0,42

0,30

0,39

0,35

Continua nella pagina successiva. 
Tabella 4. Continua dalla pagina precedente.

Mais da insilato (Stone e Hilborn, 2012)

Mais da insilato che segue mais o cereali a paglia, aratura primaverile 0,3

Mais da insilato che segue cereali a paglia, aratura estiva 0,33

Mais da insilato che segue cereali a paglia, con 10\% residui $\quad 0,23$

Mais da insilato che segue cereali a paglia, con 30\% residui $\quad 0,12$

Mais da insilato che segue colture a righe, aratura primaverile 0,3

$\begin{array}{ll}\text { Mais da insilato che segue colture a righe, aratura estiva } & 0,34\end{array}$

$\begin{array}{ll}\text { Mais da insilato che segue colture a righe, } 10 \% \text { residui } & 0,21\end{array}$

$\begin{array}{ll}\text { Mais da insilato che segue colture a righe, } 30 \% \text { residui } & 0,17\end{array}$

Mais da insilato che segue colture a righe, con applicazione primaverile di letame $\quad 0,24$

$\begin{array}{ll}\text { Mais da insilato che segue foraggera, aratura primaverile } & 0,18\end{array}$

$\begin{array}{ll}\text { Mais da insilato che segue foraggera, aratura estiva } & 0,20\end{array}$

Mais da insilato con cover crop invernale che segue cereali a paglia, aratura primaverile $\quad 0,26$

Mais da insilato con cover crop invernale che segue colture a righe, aratura primaverile $\quad 0,24$

Mais da insilato con cover crop invernale che segue colture a righe, 10\% residui 0,17

Mais da insilato con cover crop invernale che segue colture a righe, con applicazione primaverile di letame 0,16

Mais da insilato con cover crop invernale che segue foraggera, aratura primaverile 0,14

Mais da insilato con file intervallate di loiessa, che segue mais o patata (e similari) 0,19

Mais da insilato con file intervallate di loiessa, che segue mais con file intervallate di loiessa 0,12

Mais da insilato con file intervallate di loiessa, che segue cereali a paglia o soia 0,17

Mais da insilato con file intervallate di loiessa, che segue mais con file intervallate di loiessa 0,09

Mais da insilato semina senza lavorazione su cover-crop invernale 0,11

Mais da insilato semina senza lavorazione su sodo ben affermato 0,05

$\begin{array}{ll}\text { Mais da insilato semina senza lavorazione su sodo, secondo anno } & 0,15\end{array}$

$\begin{array}{ll}\text { Mais da insilato semina senza lavorazione su sodo, dopo secondo anno } & 0,18\end{array}$

Mais da granella (Stone e Hilborn, 2012)

Mais da granella che segue mais o cereali a paglia, aratura primaverile $\quad 0,21$

$\begin{array}{ll}\text { Mais da granella che segue mais o cereali a paglia, aratura estiva } & 0,28\end{array}$

Mais da granella che segue mais o cereali a paglia, con 10\% residui $\quad 0,15$

$\begin{array}{ll}\text { Mais da granella che segue mais o cereali a paglia, con 30\% residui } & 0,08\end{array}$

$\begin{array}{ll}\text { Mais da granella che segue colture a righe, aratura primaverile } & 0,18\end{array}$

$\begin{array}{ll}\text { Mais da granella che segue colture a righe, aratura estiva } & 0,24\end{array}$

$\begin{array}{ll}\text { Mais da granella che segue colture a righe, } 10 \% \text { residui } & 0,10\end{array}$

$\begin{array}{ll}\text { Mais da granella che segue colture a righe, } 30 \% \text { residui } & 0,07\end{array}$

Mais da granella che segue colture a righe, con applicazione primaverile di letame $\quad 0,13$

$\begin{array}{ll}\text { Mais da granella che segue foraggera, aratura primaverile } & 0,11\end{array}$

$\begin{array}{ll}\text { Mais da granella che segue foraggera, aratura estiva } & 0,18\end{array}$

Girasole-barbabietola-tabacco-pomodoro (Rousseva, 2004)

Girasole $\quad 0,32$

$\begin{array}{ll}\text { Barbabietola } & 0,34\end{array}$

$\begin{array}{lr}\text { Tabacco } & 0,71\end{array}$

Orticole

Lenticchia (Sonneveld e Nearing, 2003) $\quad 0,39$

$\begin{array}{ll}\text { Fagioli (Stone and Hilborn, 2000) } & 0,5\end{array}$

$\begin{array}{ll}\text { Pisello (Sonneveld e Nearing, 2003) } & 0,32\end{array}$

Pomodoro (Sonneveld e Nearing, 2003)

Altre orticole (Neitsch et al., 2000)

$\begin{array}{lr}\text { Orticole miste dopo orticole miste } & 0,5\end{array}$

$\begin{array}{lr}\text { Orticole miste dopo cover crop invernale } & 0,42\end{array}$

$\begin{array}{ll}\text { Cavolo dopo colture a righe } & 0,29\end{array}$

$\begin{array}{ll}\text { Cavolo con cover crop invernale dopo colture a righe } & 0,25\end{array}$

$\begin{array}{ll}\text { Cavolo con aratura primaverile o con residui dopo foraggera } & 0,16\end{array}$

Cavolo con aratura primaverile o con residui o con cover crop invernale dopo foraggera $\quad 0,24$

$\begin{array}{ll}\text { Cavolo dopo foraggera con aratura estiva } & 0,24\end{array}$

$\begin{array}{ll}\text { Cavolo dopo cover crop che segue foraggera, aratura estiva } & 0,18\end{array}$

$\begin{array}{lr}\text { Rape dopo patata (e similari) } & 0,30\end{array}$

$\begin{array}{ll}\text { Rape dopo cover crop invernale che segue patata (e similari) } & 0,26\end{array}$

$\begin{array}{ll}\text { Meloni/angurie dopo foraggera, aratura estiva } & 0,2\end{array}$

Meloni/angurie dopo foraggera o cover crop invernale, aratura primaverile $\quad 0,16$

$\begin{array}{ll}\text { Meloni/angurie dopo meloni/angurie con cover crop invernale } & 0,24\end{array}$

$\begin{array}{ll}\text { Meloni/angurie con } 10 \text { di residui, dopo meloni/angurie pacciamati } & 0,17\end{array}$

Piantagione in sequenza di lattuga, dopo cover crop di loiessa, aratura primaverile $\quad 0,16$

Carote dopo foraggera, aratura estiva

Continua nella pagina successiva. 
Tabella 4. Continua dalla pagina precedente.

Altre orticole (Neitsch et al., 2000)

Carote dopo cover crop che segue foraggera, aratura estiva

Carote dopo cereali a paglia, aratura estiva

Carote dopo cereali a paglia, aratura primaverile

Cipolle dopo cover crop che segue cereali a paglia, aratura primaverile

0,46

Cipolle dopo cover crop che segue cereali a paglia, aratura primaverile e $30 \%$ di residui

Foraggere

$1^{\circ}$ anno - Foraggera, semina diretta primaverile 0 estiva su quantità elevata di residui colturali

0,26

$1^{\circ}$ anno - Foraggera, semina diretta primaverile su bassa quantità di residui colturali

$1^{\circ}$ anno - Foraggera, semina diretta primaverile su quantità elevata di residui colturali

$2^{\circ}$ anno e successivi foraggera (se ben sviluppata)

Medica dopo $2^{\circ}$ anno

vetta (Sonneveld e Nearing, 2003)

Favetta (da erbaio, da granella o da sovescio) semina autunnale

to stabile (Sonneveld e Nearing, 2003)

Prato stabile misto in ottimo stato

Piante da fibra (Sonneveld e Nearing, 2003)

Lino

ratiche conservative (gestionali) del suolo (P)

(Stone e Hilborn, 2012)

Lavorazione e semina a rittochino

Lavorazione e semina in traverso

Aratura estiva-autunnale e successiva preparazione del letto di semina con amminutamento del suolo

Aratura primaverile e preparazione del letto di semina con amminutamento del suolo

Lavorazione con ripuntatore 0 altri strumenti conservativi (chisel)

Semina su residui (mulch tillage)

Presenza di drenaggio tubolare profondo

Coltivazione a strisce non livellari (in traverso)

Coltivazione con semina sui culmi dell'assolcatura dell'anno precedente (ridge tillage)

Semina dopo discatura

Colture alternate fra le righe (intercropping)

Colture alternate fra le righe copertura densa (intercropping)

Semina mediante disco assolcatore (zone tillage)

Semina su sodo

Applicazione artificiale di mulch (paglie, ecc.)

lizzando il modello RUSLE, modificando il fattore C (copertura del suolo dovuto alla coltura) e attribuendo un valore adeguato al fattore $\mathrm{P}$ ( pratiche gestionali finalizzate alla protezione del suolo).

Il valutatore indipendente potrà valutare l'efficacia degli impegni della M10 utilizzando i due suddetti modelli (1) e (2) facendo variare il fattore $\mathrm{C}$ (fattore Copertura vegetale) in relazione ad una coltura praticata, A tal fine, si suggerisce di operare come segue:

Si applicheranno le due equazioni: Fattuale (F) e Controfattuale (CF) in ambiente GIS ai poligoni del Land Parcel System che identificano le aree a seminativo a rischio di erosione e per le quali il beneficiario del pagamento unico ha l'obbligo di applicazione dello standard 1,1di condizionalità e per le quali ha aderito alla M10 con impegni che modificano la copertura del suolo (fattore $\mathrm{C}$ ) o la gestione del suolo, In questo modo si otterrà il valore del rischio di erosione in condizioni $\mathrm{F}$ e CF.

Il risultato delle due equazioni verrà diviso per 0,12 (che è il valore del fattore $\mathrm{C}$ utilizzato per ricavare l'erosione media annua sulle 60 aree campione), ottenendo il valore dell'erosione in assenza di vegetazione nelle due condizioni $\mathrm{F}$ e $\mathrm{CF}$.

I valori così ottenuti verranno moltiplicati per il fattore colturale $\mathrm{C}$ appropriato per la coltura in esame e per il fattore $\mathrm{P}$ (pratiche conservative), In assenza di misure sperimentali dirette dei valori da attribuire ai fattori $\mathrm{C} \mathrm{e} \mathrm{P}$ si potrà fare riferimento ai valori riportati in Tabella 4 , desunti dalla letteratura scientifica internazionale.

\section{Bibliografia}

Bazzoffi P, 2015. Measurement of rill erosion through a new UAV-GIS methodology. Ital. J. Agron. 10(s1):708.

Bazzoffi P, Ciancaglini A, Laruccia N, 2011. Effectiveness of the GAEC cross compliance standard Short-term measures for runoff water control on sloping land (temporary ditches and grass strips) in controlling soil erosion. Ital. J. Agron. 6(s1):e3.

Bazzoffi P, Francaviglia R, Neri U, Napoli R, Marchetti A, Falcucci M, Pennelli B, Simonetti G, Barchetti A, Migliore M, Fedrizzi M, Guerrieri M, Pagano M, Puri D, Sperandio G, Ventrella D, 2015. Efficacia ambientale degli Standard di condizionalità 1.1a (Solchi acquai temporanei) e $1.2 \mathrm{~g}$ (Copertura vegetale durante tutto l'anno nei seminativi ritirati dalla produzione) nella riduzione dell'erosione del suolo e valutazione del differenziale economico di competitività a carico delle aziende agricole. Ital. J. Agron. 10(s1):710.

Desmet P. J. J., Govers G. 1996. A GIS procedure for automatically calculating the USLE LS factor on topographically complex landscape units, J. Soil and Water Cons., 51(5), 427-433.

Gobin A, Govers G, Kirkby MJ, Le Bissonnais Y, Kosmas C, Puigdefabregas J, Van Lynden G, Jones RJA, 1999. PESERA Project Technical Annex, European Commission.

Mitasova H, Mitas L, Brown BM, Gerdes DP, Kosinovsky I, 1995. 
Modeling spatially and temporally distributed phenomena: new methods and tools for GRASS GIS. Int. J. Geogr. Inf. Sci. 9:443-446.

Moore I, Burch G, 1986. Physical basis of lenght-slope factor in the universal soil loss equation. Soil Sci. Soc. Am. J. 50:1294-1298.

Neitsch SL Arnold JG, Kiniry JR, Srinivasan R, Wiliams JR, 2002. Soil and w assessment tool user's manual, ver. 2000. GSWRL Report 0202, BRC Report 02-06, TR-192. Texas Water Resources Institute, College Station, TX, USA.

OCSE, 2001. Environmental indicators for agriculture. Methods and results. Agriculture and Food. Vol. 3. OECD, Paris, France.

Renard KG, Foster GR, Weessies GA, Mccool DK, Yoder DC, 1997. Predicting soil erosion by water: a guide to conservation planning with the Revised Universal Soil Loss Equation (RUSLE). Agriculture Handbook 703, US Department of Agriculture.

Rousseva S, 2004. Ideas for physical interpretation of the USLE. In: D.M. Gabriels, D.R. Ghirardi, I. Nielsen, S. Pla and E.L. Skidmore (eds.) Invited Presentations, College on Soil Physics. ICTP Lecture notes 18:309-320. http//users.ictp.it/pub_off/lectures/ns018/26R ousseval.pdf

Schertz DL, Nearing MA, 2005. Erosion tolerance/soil loss tolerance. In: R. Lal. (ed.) Encyclopedia of Soil Science. CRC Press, Boca Raton, FL, USA, pp 640-2.

Sonneveld B, Nearing M, 2003. A nonparametric/parametric analysis of the Universal Soil Loss Equation. Catena 52:9-21.

Stone PR, Hilborn D, 2012. Universal Soil Loss Equation (USLE). Factsheet Agdex \#572/751. Ministry of Agriculture, Food and Rural Affairs, Ontario, Canada. Sito web: http:/www.omafra.gov.on.ca/ english/engineer/facts/12-051.htm

Wischmeier WC, Smith DD, 1978. Predicting rainfall erosion losses - A guide to conservation planning. Agricultural Handbook No. 537. US Department of Agriculture, Washington, DC, USA. 